JAIME FRANCISCO GÓMEZ GÓMEZ

UNIVERSIDAD DE GUADALAJARA

GUADALAJARA, MÉXICO

JAIME.GOMEZ@CUAAD.UDG.MX

FRANCISCO JAVIER GONZÁLEZ MADARIAGA

UNIVERSIDAD DE GUADALAJARA

GUADALAJARA, MÉXICO

FRANCISCO.MADARIAGA@CUAAD.UDG.MX

LUIS ALBERTO ROSSA SIERRA

UNIVERSIDAD PANAMERICANA

GUADALAJARA, MÉXICO

LUROSA@UP.EDU.MX
Fecha de recepción: 29/08/2018

Fecha de aceptación: 28/06/2019

Cómo citar: Gómez Gómez, JF. et al (2019)

Nuevos materiales a partir de residuos

textiles: una perspectiva del diseño industrial

RChD: creación y pensamiento, 4 (7), 1-12

DOI: 10.5354/0719-837X.2019.49872

Revista Chilena de Diseño,

RChD: creación y pensamiento

Universidad de Chile

2019, 4(7)

http://rchd.uchile.cl

\section{Nuevos materiales a partir de residuos textiles: una perspectiva del diseño industrial}

\author{
New Materials From Scrap Denim: \\ An Industrial Design Perspective
}

Resumen. El presente trabajo es una breve descripción de algunas experiencias efectuadas por el grupo de investigación Innovación Tecnológica para el Diseño del Centro Universitario de Arte Arquitectura y Diseño de la Universidad de Guadalajara a través de proyectos terminales de diseño industrial. Una de las áreas de interés de este grupo de investigación es el trabajo con residuos de algunas empresas manufactureras de la localidad para proponer nuevos materiales a partir de dichos residuos, desarrollando investigación sobre fibras de agave, textiles, residuos plásticos y basalto por mencionar algunos en los que se está trabajando. Por otro lado, es importante destacar la participación de los estudiantes tanto en la elaboración del material como de propuestas de productos a partir de dichos materiales. Esto representa una oportunidad para, por un lado, adentrarlos en el tema de los procesos de manufactura para la elaboración de prototipos, y por otro, acercarlos sobre la marcha al conocimiento de las propiedades y atributos de los materiales. Si bien el objetivo de estas actividades no es desarrollar experiencias didácticas, sino resolver una problemática dentro de un sector productivo concreto, este último aspecto resulta de gran interés dado que de manera formal muchos de ellos, por el tipo de diseño curricular, no recibieron este tipo de formación de carácter técnico.

Palabras clave: diseño industrial, materiales compuestos, revaloración de residuos, suprareciclaje.

Abstract. The present work is a brief description of a few experiences performed by this research group, Technological Innovation for Design, with the collaboration of students working their terminal projects at Centro Universitario de Arte Arquitectura y Diseño from the University of Guadalajara. One of the interest areas of this group is to work with scraps from local manufacturing companies in order to develop and propose new materials and products using them, focusing research in agave fibers, textiles, plastics and basalt, just to mention a few in which work is currently being done. This is an opportunity, in one hand, for students to have a deeper knowledge of manufacturing processes, and on the other, to give them an approach to the knowledge of materials properties and attributes. Nevertheless the aim of the project is not a didactic experience, this last aspect is of great interest since, formally, many of these students, due to the type of design curriculum they had, did not receive this type of technical training.

Keywords: composite materials, industrial design, revaluation of waste, scrap upcycling. 


\section{Introducción}

El creciente interés de los diseñadores por desarrollar nuevos materiales a partir de residuos de los procesos de manufactura se ha constituido como una tendencia global. En este contexto se ha observado que las aportaciones a este fenómeno, asociadas en gran medida al Do it Yourselfo DIY (Ayala-García, Rognoli \& Karana, 2017), varían de acuerdo al enfoque y tipo de experimentación, con o sin soporte formal de laboratorio de por medio. No obstante, los pioneros en trabajar con residuos para su aprovechamiento fueron Braungart y McDonough (2003) y, con un planteamiento metodológico más riguroso, plantearon la idea de utilizarlos como insumo ("alimento") para otro proceso de la propia empresa lo que logró su revalorización, es decir, supraciclarlos (en inglés upcycling). Actualmente son muchas las empresas manufactureras que hacen lo opuesto, es decir, desechan los residuos sin explorar la posibilidad de aprovecharlos en otros productos, desvalorizándolos. En algunos casos, los residuos que permanecen en el área de trabajo se utilizan para la elaboración de productos con una vida útil muy corta (downcycling), como es el caso del patronaje de los pantalones de mezclilla (nombre que se le da al textil que en inglés se conoce como denim) o jeans, o simplemente no se utilizan de manera alguna, como es el caso del residuo de los productos de basalto de la población artesanal de San Lucas Evangelista, del estado de Jalisco. Con base en la idea de la revalorización de los residuos, con el apoyo de estudiantes en el proceso de realización de su proyecto terminal, este grupo de investigación abordó el problema del residuo de patronaje de mezclilla de una empresa manufacturera de la ciudad de Guadalajara, México. Como objetivo general, se buscó verificar la factibilidad de reutilizar los desechos de retazos de mezclilla para su revalorización. Como objetivos particulares, se buscó: desarrollar materiales compuestos de matriz polimérica a partir de desechos industriales textiles de preconsumo de mezclilla y llevar a cabo su transformación mediante los procesos ya utilizados convencionalmente en los materiales de partida sin cargas o refuerzos.

Cabe mencionar que durante el proceso se observó que, a pesar de las implicaciones técnicas en la fabricación y transformación del nuevo material, el aspecto estético-simbólico que ha permitido la apropiación de la mezclilla por parte del consumidor global (Miller \& Woodward, 2007) fue factor determinante para el diseño de dichos productos (Gómez, et al., 2016).

\section{El caso de los residuos de patronaje de pantalones de mezclilla}

La empresa de la cual surge el presente proyecto tiene un desperdicio del $15 \%$ de un tramo de $1.3 \mathrm{~m}$ de tela para la confección de un pantalón. Semanalmente produce 1600 pantalones, desperdiciando $312 \mathrm{~m}$ en dicho periodo, y si se consideran las cincuenta y dos semanas laborales, esto constituye un desperdicio de $16.224 \mathrm{~m}$ lineales anuales. En la actualidad, los sobrantes del patronaje son recolectados para la fabricación de borlas para pulidoras orbitales, que tienen una vida útil muy corta y un valor de cambio muy bajo, por lo que se desechan rápidamente al vertedero. Esta solución, si bien contribuye a atender el problema inmediato de la acumulación de material de desecho dentro de la empresa, no agrega valor a dicho material, sino que lo devalúa, y a dicho proceso se le denomina como infraciclaje. Una aproximación desde el supraciclaje permite agregar valor a un material que desde el inicio no se considera útil. Con el objetivo de revalorizar las fibras, en este escrito se reportan algunas experiencias de investigación acerca del proyecto que consiste en la 

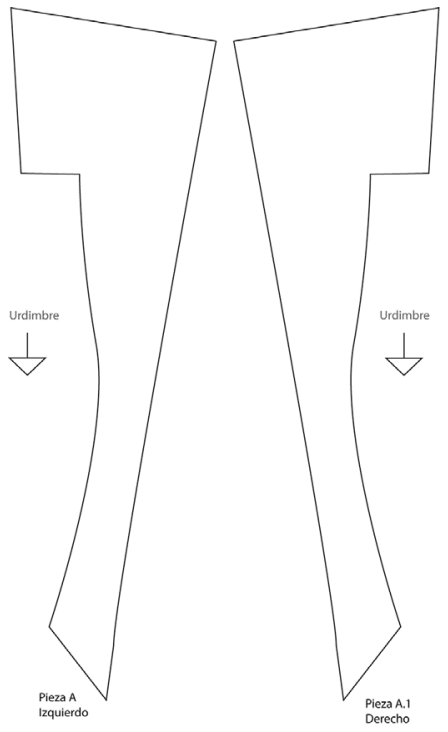

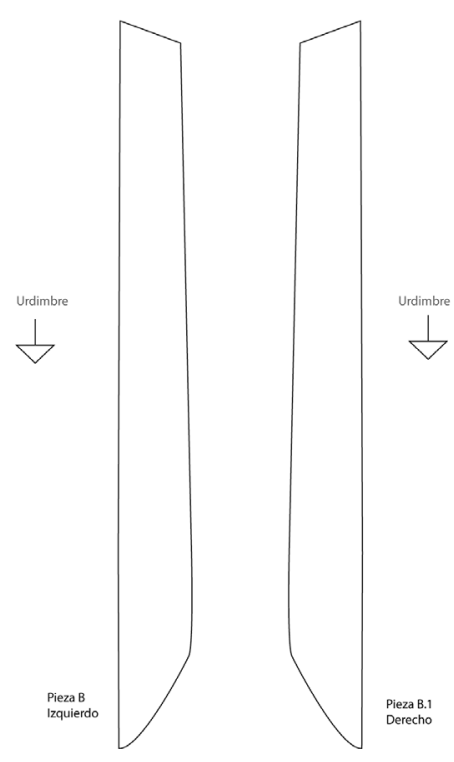

Figura 1. Muestras de retazos sobrantes regulares de patronaje. Fuente: elaboración propia.

elaboración de un material compuesto, a base de mezclas de este material textil deshilado como carga en una matriz polimérica. El textil utilizado por esta empresa es de tejido plano con ligamento tipo sarga, estaba constituido en la trama con fibras $22 \%$ de poliéster y $2 \%$ de elastano, y $76 \%$ en la trama. Anteriormente, en una primera aproximación al trabajo se buscó elaborar un material a base de este textil deshilado aglutinado con un adhesivo (Rosa, et al., 2015). Paralelamente, se procedió a clasificar los retazos del patronaje para evaluar la posibilidad de unirlos mediante una costura ordinaria y con ello formar lienzos nuevos para la elaboración productos a partir de los propios procesos de la empresa. En la figura I se muestran dos tipos de retazos y su posición respecto a la urdimbre del textil.

Se detectaron cuatro clases de retazos regulares con los que se elaboraron ocho tipos distintos de muestras de confección. Un aspecto importante de este procedimiento es que los estudiantes involucrados en este proceso constataron que, a pesar de la simetría del corte de las piezas, en función de su posición respecto a la urdimbre de la tela, hay direccionalidad en el retazo resultante, y eso afecta la costura final, dado que el lienzo puede dejar de ser plano. Con todo y ello, esta estrategia resultó ser del interés del fabricante, ya que al no requerir de nuevos procesos o tecnologías procedió a modificar la distribución de los patrones en la línea de corte para obtener figuras más regulares para de esta forma facilitar el proceso de unión de los nuevos lienzos. Por su parte, a partir de los resultados de esa primera aproximación de mezclas de material deshilado con aglutinantes, en una segunda etapa se trabajó con dos combinaciones multicapa de mezclilla con polipropileno (PP), que es uno de los polímeros termoplásticos más utilizados a nivel mundial y cuyo desecho tiene el potencial para ampliar su vida útil. Posteriormente, con este mismo polímero termoplástico, se retomaron las mezclas del textil deshilado con esta matriz polimérica. Con el objeto de conocer cómo las fibras afectan el módulo de elasticidad y el porcentaje de alargamiento del material resultante, se efectuaron ensayos de flexión y de tracción de las tres combinaciones, así como la medición de sus densidades aparentes, concluyéndose que dichas propiedades se ven disminuidas respecto al 
Figura 2. Muestra de corte con láser en compuesto PP-mezclilla deshilada (fotografía: Eric Nápoles).

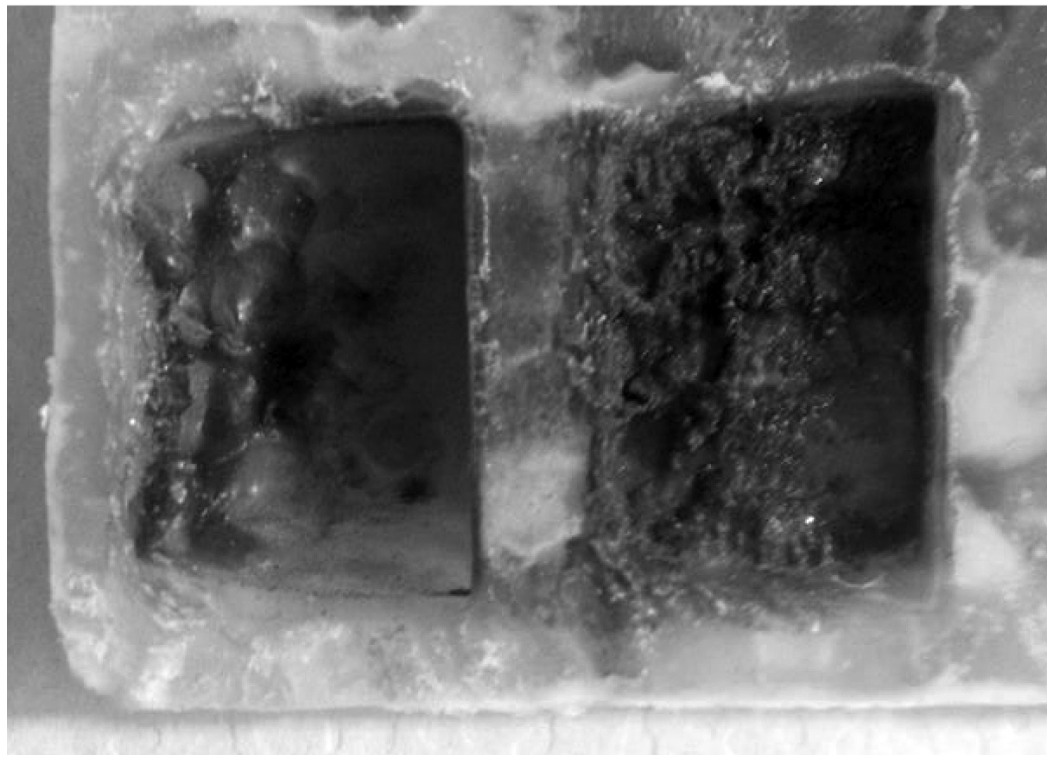

polímero de partida (Gómez, González, Rosa \& Abt, 2016), esto es atribuible al contenido de poliéster y elastano. A pesar de ello, los nuevos materiales presentan ventajas respecto a otros reciclados o reutilizados (cartón, lona, neopreno), entre las que destacan la rigidez, impermeabilidad y la apariencia atractiva. Si se parte de este conocimiento, en esta tercera etapa se trabajó con dos grupos de estudiantes en proyecto terminal de la Licenciatura en Diseño Industrial, con el objeto de valorar la posibilidad de diseñar y producir productos de consumo con dichas combinaciones de materiales.

El trabajo con estudiantes se desarrolló en seis fases: 1) evaluación de propiedades; 2 ) estudio del estado de la cuestión respecto a materiales compuestos similares; 3) elaboración de muestras del nuevo material; 4) detección de necesidades de usuarios; 5) pruebas de procesos de manufactura con el nuevo material; y 6) diseño y fabricación de productos de consumo. Es importante comentar que las pruebas de procesos incluyeron corte con láser y corte con router $\mathrm{CNC}$. Por tratarse de un polímero semicristalino de relativamente bajo punto de fusión, se observó que el corte con láser no fue apropiado para el trabajo con este material (ver figura 2) mientras que los mejores resultados se observaron con el corte con router CNC. Los resultados de este proceso de diseño fueron reportados por Gómez, González, Rosa y León (2016).

Para la cuarta etapa, y tomando en consideración estas observaciones, se optó por trabajar con una mezcla de estas fibras textiles con polimetacrilato de metilo (PMMA) que, por un lado, presenta mayor rigidez que el PP y por otro, por su transparencia y excelentes propiedades ópticas, permite lograr distintos motivos que pueden ser visualmente atractivos para el diseño de productos. Si bien el proceso de fabricación y reciclado de láminas de PMMA es por métodos químicos, es posible conseguir reciclar el material con menor pérdida de sus propiedades respecto a otros polímeros termoplásticos que se van degradando a consecuencia del reciclaje térmico (Brydson, 2000). De esta forma, es posible alargar la vida útil de ambos componentes en un nuevo material y, consecuentemente, en un nuevo producto. 


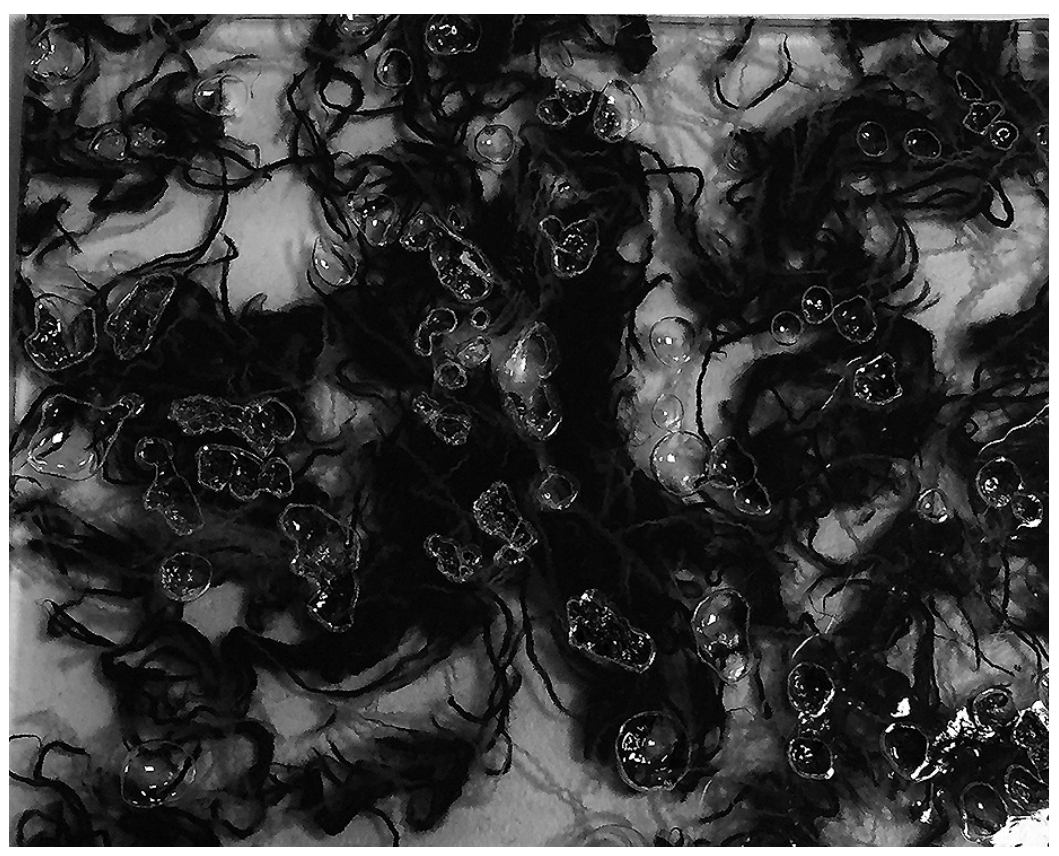

El proyecto se desarrolló en cinco fases que a continuación se enumeran: 1) preparación de muestras y fabricación de lámina, 2) investigación del estado de la técnica, 3) investigación bibliográfica de productos y soluciones existentes, 4) pruebas de procesamiento, 5) conceptualización de propuestas y construcción de prototipos funcionales.

\section{Sobre la preparación de muestras de PMMA}

En un primer intento de elaboración del material compuesto de fibra de mezclilla con PMMA se observó que el material textil mezclado con la matriz polimérica, tanto en retazo como deshilado, generó defectos en el compuesto resultante (ver figura 3). Esto se debe a que las fibras textiles atrapan humedad y/o aire, para propiciar la formación de burbujas durante el proceso de vaciado de la resina en proceso de polimerización in situ. Cada burbuja, tanto superficial como interna, es un concentrador de tensiones, por lo que no es posible asegurar uniformidad en las respuestas mecánicas del material resultante, así como su calidad final.

Con el apoyo de un nuevo grupo de estudiantes en proyecto terminal de la Licenciatura en Diseño Industrial, se llevaron a cabo distintas preparaciones para la impregnación de las fibras textiles con sustancias compatibles con la matriz de PMMA. De dichas preparaciones se seleccionó aquella que permitió lograr un material laminado con fibras embebidas sin presentar burbujas o defectos superficiales.

Por razones de trámite de patente, en este documento se omite la descripción de dicho procedimiento. Cabe mencionar que de la información obtenida y analizada del estado de la técnica se pudo tomar la decisión de llevar a cabo dicha solicitud. Es importante aclarar que la caracterización mecánica de este material compuesto está por realizarse. Se contemplan ensayos a tracción y a flexión en tres puntos, aunque está por definirse bajo qué modo podrían efectuarse los ensayos de impacto.
Figura 3. Muestra de lámina defectuosa de mezclillaPMMA (fotografía: Jaime Fco. Gómez) 
Figura 4. Panel de conceptos de algunos productos elaborados con fibras de pre y posconsumo (elaborado por estudiantes).

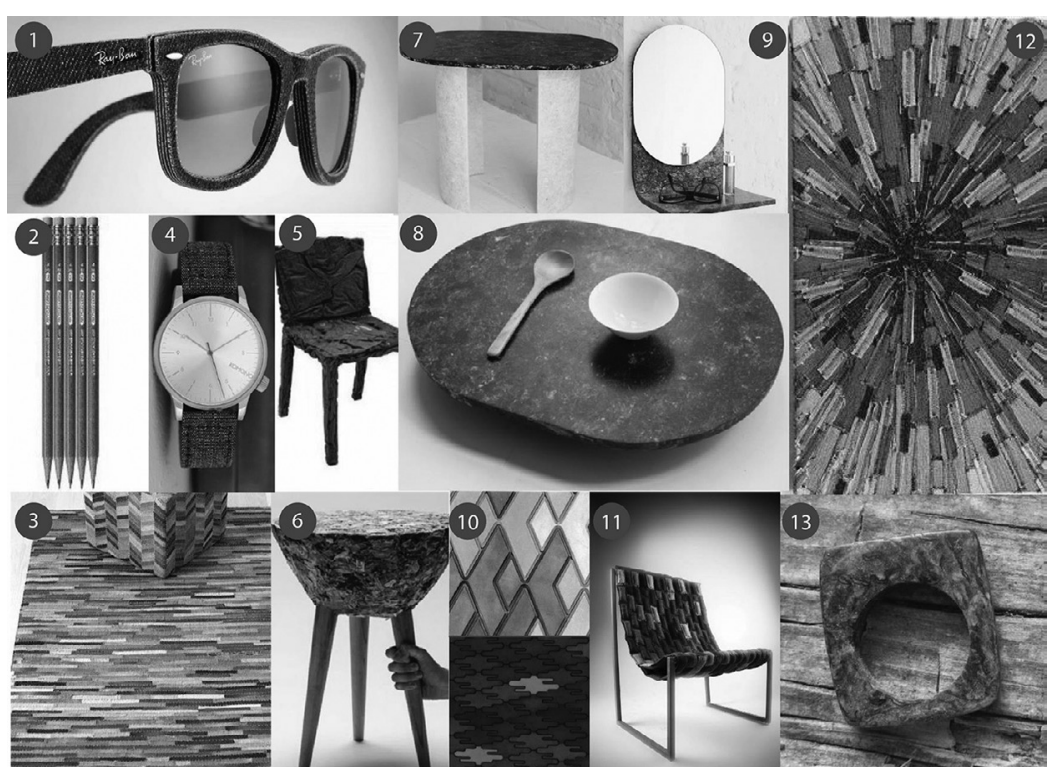

\section{Proceso de diseño y pruebas}

Toda vez que se logró desarrollar un material laminado de $3 \mathrm{~mm}$ con superficies lisas y libre de burbujas en su interior, se procedió a trabajar con dos equipos de diseñadores industriales con el objeto de diseñar propuestas de productos. Paralelamente a la preparación de las muestras, ambos equipos efectuaron una revisión de productos elaborados con estas fibras textiles y otros aglutinantes y se procedió a clasificarlos por área de aplicación. Parte de los hallazgos de esta etapa se sintetizan en el panel de conceptos de la figura 4. Se observa que muchos de lo que en la actualidad se elabora a partir de estos residuos de pre y posconsumo mantiene la apariencia del textil, pero en su mayoría son aglomerados opacos que solo en algunos de los casos han sido caracterizados. También se observó que algunos productos se fabricaron a partir del reprocesamiento de las fibras para hilar nuevos rollos de tela, lo que implica la separación de las fibras de algodón, que son susceptibles de hilarse nuevamente, de las fibras sintéticas como el poliéster y el elastano, que son difíciles de hilar.

Además de lo que se presenta en el panel de conceptos, se encontraron aplicaciones en el área de la construcción, más por sus propiedades técnicas (baja conductividad térmica, resistencia a la humedad, al fuego o al crecimiento de hongos, por mencionar algunas) que por sus propiedades estéticas. Derivado de este primer acercamiento se identificaron algunas áreas potenciales de aplicación, las cuales se presentan en la tabla 1.

\begin{tabular}{|c|c|c|c|c|}
\hline \multicolumn{5}{|c|}{ Áreas potenciales de aplicación } \\
\hline $\begin{array}{l}\text { Accesorios } \\
\text { personales }\end{array}$ & $\begin{array}{l}\text { Accesorios decora- } \\
\text { tivos para el hogar }\end{array}$ & $\begin{array}{l}\text { Accesorios de } \\
\text { oficina }\end{array}$ & $\begin{array}{l}\text { Identidad de } \\
\text { marca y packaging }\end{array}$ & $\begin{array}{l}\text { Display para } \\
\text { punto de venta }\end{array}$ \\
\hline $\begin{array}{l}\text { Zapatos, } \\
\text { lentes relojes, } \\
\text { accesorios para } \\
\text { el cabello, joyería, } \\
\text { bolsos y carteras, } \\
\text { personalización } \\
\text { de gadgets, } \\
\text { relojes }\end{array}$ & $\begin{array}{l}\text { Mobiliario, } \\
\text { mamparas, } \\
\text { accesorios para baño, } \\
\text { cancelería, manerales } \\
\text { y herrajes, luminarias } \\
\text { para interiores y } \\
\text { exteriores, espejos, } \\
\text { biombos }\end{array}$ & $\begin{array}{l}\text { Forro para } \\
\text { agenda o } \\
\text { cuadernos, } \\
\text { plataformas } \\
\text { para laptop, } \\
\text { portafolios, } \\
\text { maletín de mano, } \\
\text { organizadores }\end{array}$ & $\begin{array}{l}\text { Estantería, } \\
\text { anuncios } \\
\text { luminosos, } \\
\text { accesorios para } \\
\text { escaparates, } \\
\text { estuches, envase } \\
\text { para productos } \\
\text { de lujo }\end{array}$ & $\begin{array}{l}\text { Anuncios } \\
\text { luminosos, } \\
\text { cubiertas, } \\
\text { exhibidores y } \\
\text { plafones }\end{array}$ \\
\hline
\end{tabular}

Tabla 1. Clasificación de usos y aplicaciones potenciales de materiales de fibras de mezclilla con diversos aglutinantes 

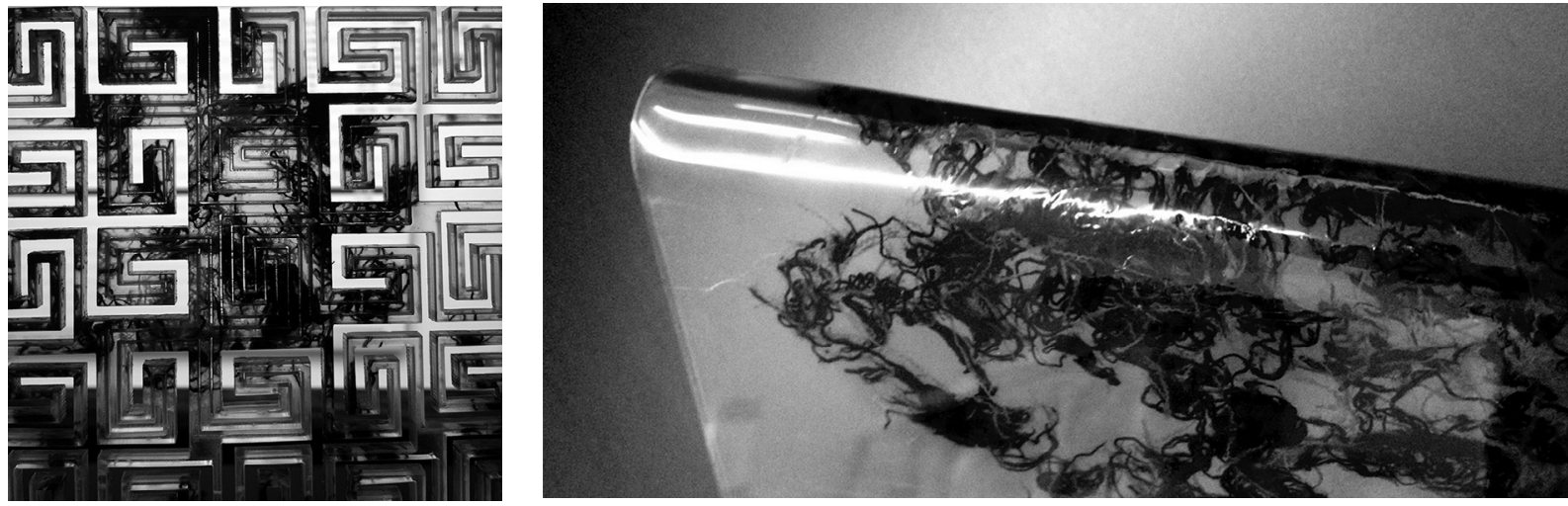

Para la siguiente etapa, los tesistas llevaron a cabo algunas pruebas de procesamiento del material con el objeto de conocer cómo se comportaría el compuesto durante su transformación: corte con router y con láser, barrenado, termoconformado, doblado y pegado. Este tipo de pruebas permitió definir los criterios de manufactura para el desarrollo de las propuestas. Respecto al corte con láser, en una primera aproximación al proyecto, se pensó en diseñar productos a partir de superficies de doble curvatura y se observó que, a pesar de la presencia de las fibras textiles, la lámina de mezclilla-PMMA aún es sensible a las entallas, por lo que se optó por trabajar con este material de la forma convencional, es decir, plano, sin someterlo a esfuerzos o, en su defecto, con curvatura en una sola dirección; no obstante, no se descarta del todo la posibilidad de continuar experimentando con este tipo de superficies. Por otro lado, se observó que, en algunos casos, el corte con láser no fue eficaz en zonas con mayor concentración de fibras (ver figura 5, izquierda), aunque esto puede ser atribuible a la calibración del equipo, dado que en algunas otras pruebas y en los prototipos finales no se observó dicho fenómeno. A su vez, se valoró la conveniencia de hacer cortes con esquinas redondeadas para evitar la propagación de grietas en caso de ser sujeto a esfuerzos diversos.

En el caso del termoconformado, se observó que el material embebido se deformaba de manera distinta a la matriz polimérica, para propiciar el surgimiento de pequeños relieves o arrugas en estiramientos uniformes y regulares pero que, a mayores alargamientos y geometrías irregulares, provocaban la rotura del material. De igual forma, se observó que el doblado de la lámina con radios amplios (ver figura 5, derecha), propiciaba la aparición de arrugas como las observadas durante el termoconformado.

Con base en la investigación bibliográfica y de campo, así como los criterios de fabricación definidos, se llevó a cabo una lluvia de ideas entre las que destacan las asociaciones del material textil con los aspectos simbólicos ligados al tinte índigo de origen asiático (Chavan en Paul, 2015), así como a elementos de la naturaleza y el paisaje.

Posteriormente y a partir de las asociaciones de ideas surgidas a lo largo del proyecto, se desarrollaron conceptos individuales como se describen a continuación:

-Asociación 1: mandalas

-Asociación 2: montañas

-Asociación 3: cristales

-Asociación 4: muro de agua
Figura 5. A la izquierda, elemento al centro de la lámina que no pudo ser cortado con láser. A la derecha, aparición de arrugas al doblar la lámina con material embebido con un radio de $2.5 \mathrm{~cm}$ Fotografías: Jaime F. Gómez. 


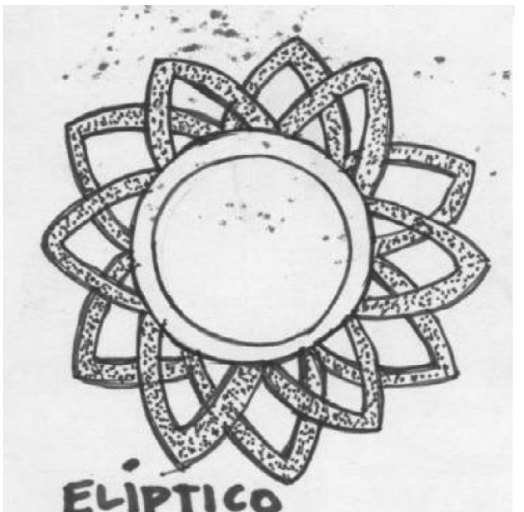

ELIPTICO

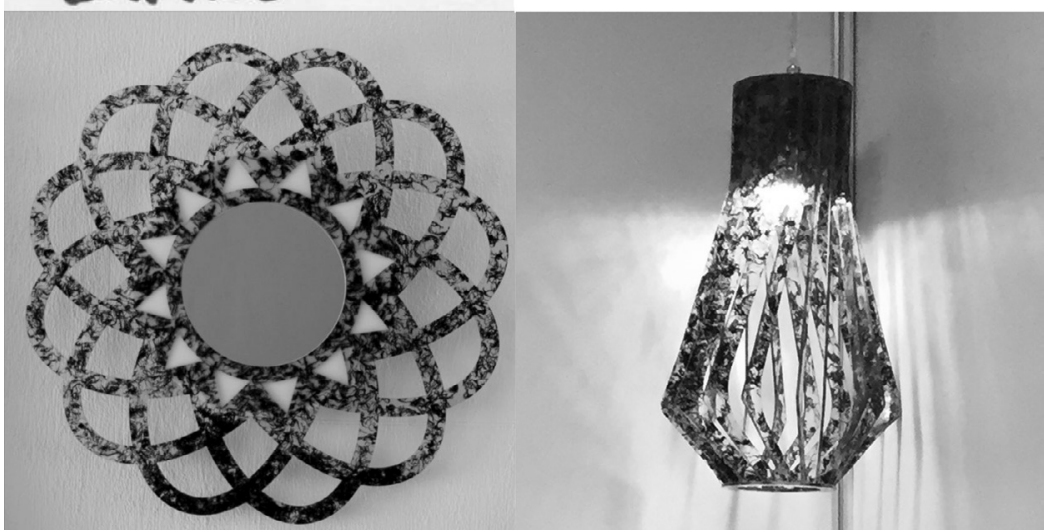

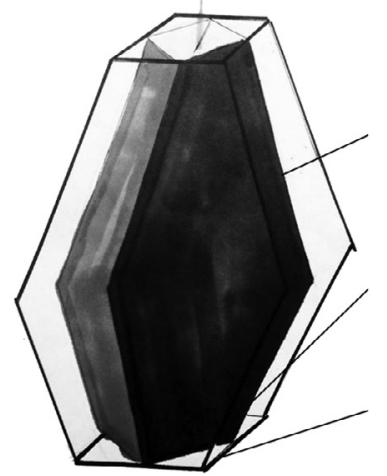

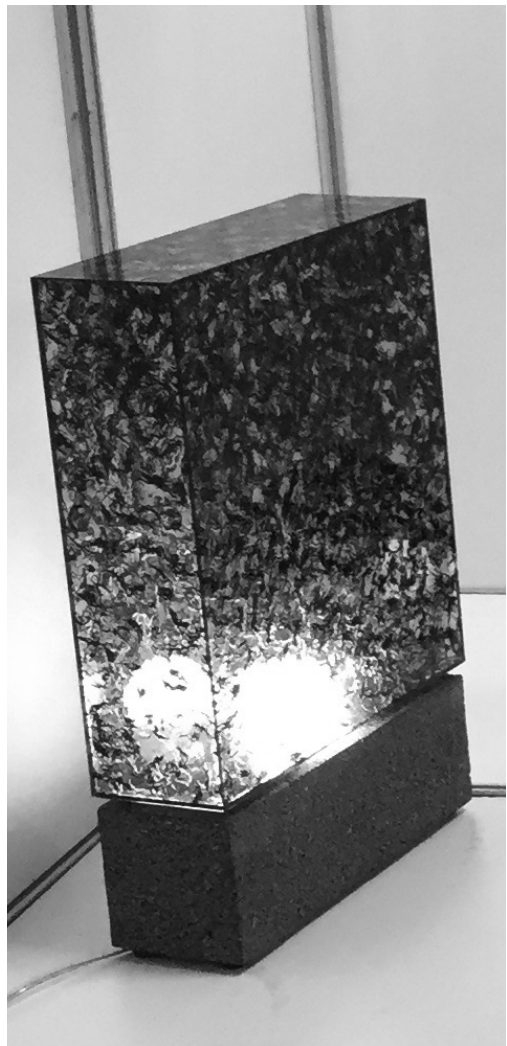

Figura 6. Bocetos de luminarias basadas en mandalas (a la izquierda) y cristales (a la derecha).

Autores y fotografías: América Díaz y Brenda Trujillo. Figura 7. Prototipo de luminaria "muro de agua". Autor y fotografía: Ricardo Juárez.
Si se considera la transparencia de la matriz y la dispersión de la fase textil, se definieron tres tipos de productos que potencializaran las cualidades estéticas y a su vez, por tratarse de objetos de un largo ciclo de vida, revalorizaran los desechos integrados en el nuevo material: luminarias, joyería y contenedores. De estas tres categorías, se presentan tres diseños de luminarias cuyos prototipos se fabricaron con las técnicas de corte con láser y router y pegado con adhesivo de cianoacrilato. Cada una corresponde a los conceptos mandala (luminaria con espejo), cristales (luminaria colgante) y muro de agua (luminaria para mesa). En la figura 6 se muestran algunos bocetos de estos conceptos. Finalmente, en el caso concreto del concepto de muro de agua, se eligió una geometría sencilla que además de utilizar el material objeto del presente desarrollo, lo combina con otro de origen volcánico pero que genera un contraste de texturas: el basalto (ver figura 7).

Por tratarse de un proyecto en proceso, queda pendiente la caracterización mecánica del nuevo compuesto a efectos de conocer en qué medida la inclusión de las fibras afecta su desempeño y valorar si dichas fibras pueden inhibir la propagación de las grietas en este material que es sensible a entallas. Por otro lado, se abre la posibilidad para desarrollar estudios con usuarios sobre asociaciones y calidad percibida de este material.

\section{Discusión}

A partir de estas experiencias, surgen algunas consideraciones en torno a los aspectos materiales de este proceso y, por otro lado, si bien la experiencia pedagógica no era el objetivo principal de este proyecto, el haber involucrado a los estudiantes de nivel terminal aportó algunas ideas respecto a la 
enseñanza de los aspectos técnicos de los materiales y la manufactura. En primera instancia refuerza el enfoque sistémico de la labor del diseñador (Jones en Metcalf, 2014) que, dada la complejidad del mundo actual, es de suma importancia ampliar. Esto es, el diseñador tenderá a formarse como un actor capaz de contribuir a la resolución de problemas de orden superior, y no solo en la escala de proyectos individuales. Los retos que presentan proyectos de esta naturaleza trascienden los métodos tradicionales de la enseñanza del Diseño, más orientados a los aspectos estéticos y expresivo, así como a la comunicación del proyecto, que a los aspectos tecnológicos y estructurales del contexto de la práctica del Diseño. Asimismo, se constata que los diseñadores tienen la capacidad de experimentar y de aprender cosas nuevas, lo cual demuestra que el Diseño Industrial es una disciplina cuyos profesionales son capaces de lograr la rápida adaptación al contexto en la práctica. Por su parte, en experiencias similares enfocadas al análisis de la economía circular en la Universidad de Leiden, Kopnina (2018), a través de varios estudios de caso con empresas elaborados por estudiantes de pregrado y posgrado, encontró que tanto en este encuadre como el cradle to cradle en la realidad no se revalorizan residuos, sino que se infraciclan, además de que la circularidad económica que se busca no es del todo posible. Señala varios aspectos que efectivamente se replican en la experiencia aquí presentada, donde se coincide en que los estudiantes involucrados, más allá del desarrollo de prototipos con el nuevo material y del aprendizaje de los aspectos técnicos inherentes al proceso de su obtención, estos son capaces de aplicar el pensamiento crítico para examinar las políticas gubernamentales a efecto de identificar posibles reformas, pero sobre todo diferenciar entre lo que es ideal y lo que es viable bajo las condiciones actuales.

En lo que respecta a los aspectos materiales se debe considerar que, en este tipo de prácticas, y como lo han señalado Bakker, Wever, Teoh \& De Clerca (2010), la propuesta cradle to cradle si bien es inspiradora y ha funcionado como base para la presente experiencia tiene algunas limitaciones. La primera de ellas, señalan, proviene de la crítica hacia la ecoeficiencia y el supuesto de adoptar la ecoefectividad, a través de la aplicación de los tres principios preconizados por la metodología (desecho equivale a alimento, utilizar el bono solar, respetar la diversidad), en la que su implementación se enfoca casi de manera exclusiva en el concepto de la equivalencia del desecho en alimento, idea con la que también coincidimos con Toxopeus, de Koeijer y Miej (2016). Para la presente experiencia resultó sencillo utilizar el desecho como alimento para otro proceso, pero la aplicación del bono solar no fue posible. De hecho, este punto es el más difícil de abordar por cualquiera que intente aplicar esta metodología dado que en función del contexto de la empresa, el bono solar por lo general queda marginado, incluso por los propios autores de dicha metodología por la atención que esta presta a la composición de los materiales. La reconversión de las fuentes energéticas en los contextos de las MIPyMEs, al menos por ahora, no es una opción viable por los elevados costos de inversión inicial que representan en este país.

En lo referente al respeto a la diversidad, desde la perspectiva del usuario, el nuevo material por sí mismo representa una paradoja dado que el textil utilizado contiene una carga de valores simbólicos que, independientemente de su procedencia geográfica, remite al reforzamiento de la identidad de sus usuarios, identidad que mantiene su carácter propio en contextos culturales específicos y distintos entre sí (Miller \& Woodward, 2007). Dichos autores 
observaron que quienes se enfrentan al tema de seleccionar sus prendas, por un lado, buscan "seguridad en la ubicuidad" y resuelven con ello el problema de la individualidad (autoafirmándose) y a su vez logran conformidad. Por ello, esta ambivalencia por el uso que se le da a este material contraviene al cuestionamiento de Braungart y McDonough a la premisa de "un tamaño acomoda a todos" (one size fits all) de las soluciones universales. Por su parte, una de las críticas más comunes que señalan Bakker, Wever, Teoh \& De Clercq es el hecho de que este tipo de acciones promueven innovaciones de carácter técnico, pero no permean hacia la sociedad o la cultura. En estas experiencias, se constató que efectivamente, fuera del contexto de la empresa, el impacto por más favorable que haya sido para las partes involucradas es poco reconocible en la sociedad y, ciertamente, las innovaciones son de carácter técnico. Respecto a las empresas, a pesar de la perspectiva prometedora del nuevo material y el método para su obtención, no fue posible conseguir un círculo virtuoso en lo concerniente al flujo de los materiales entre ellas. Asimismo, en sus conclusiones resaltan el riesgo de adoptar esta metodología con cierto dogmatismo, mientras que no siempre es factible su aplicación en el entorno de las empresas. En la presente experiencia, efectivamente, nos hemos topado con las limitaciones propias del contexto empresarial local, donde además de la conciencia del daño ambiental por parte de las empresas, es necesario trabajar en incentivos para cumplimentar en el futuro cercano nuevas normas de carácter ambiental.

Otra arista que se deberá de abordar respecto a la metodología cradle to cradle es que no necesariamente se trata de una estrategia innovadora, sino más bien una metodología optimizadora dentro de los procesos en las empresas (Toxopeus, de Koeijer \& Meij, 2015). Desde la perspectiva del diseño estratégico, esto se podrá tomar como una estrategia de innovación incremental y no de una de carácter radical.

Por su parte, con este proyecto se verifica la importancia de la colaboración entre la industria y la academia a través de la investigación y la aplicación de los hallazgos en proyectos terminales de utilidad para la empresa. Si bien no se plantea como una relación de codiseño, se aprecia la necesidad de involucrar academia con industria y a su vez hacer que la industria pueda asumir un papel más activo en este proceso, más allá del papel de proveedor pasivo de la materia objeto de investigación. La consolidación de estas relaciones tenderá a cimentar la economía circular, no obstante, las variables para tal efecto van más allá de lo descrito en estos avances de investigación.

Finalmente, la revalorización de este material y cualquier otro que se efectúe, así como los productos que con ellos se elaboren, dependerá en gran medida del uso que el usuario final le otorgue. Los ejemplos aquí mostrados se basan en el supuesto del alargamiento de la vida útil del residuo convertido en un bien de consumo relativamente duradero. Utilizar un material de estas características deberá de tomar en cuenta aplicaciones en bienes duraderos y procurar no emplearlo en artículos desechables.

Se ha conseguido otorgarle, a priori, una segunda vida a este residuo, pero solo a partir de un estudio longitudinal con los usuarios sobre la duración de los bienes diseñados se podrá determinar si estas acciones han sido efectivas. 


\section{Conclusiones}

En el presente proyecto se demostró que es factible y posible desarroIlar materiales compuestos a partir de desechos industriales textiles de preconsumo mezclados con matrices poliméricas, que alargan su vida útil y con ello se consigue su revaloración como material para diseñar y fabricar productos. A su vez, se comprobó que es posible llevar a cabo su transformación mediante los procesos ya utilizados convencionalmente en las láminas de acrílico sin cargas o refuerzos. Esto permite vislumbrar la posibilidad de diseñar y elaborar otros compuestos con fibras embebidas, tanto textiles como naturales, para uso decorativo, así como para aplicaciones funcionales y estructurales de bajo compromiso mecánico.

Es prudente señalar que quedan varios pendientes para tener una valoración más cercana sobre la viabilidad de estos materiales como acciones para el desarrollo sostenible, entre los que se destaca: hacer análisis químicos de las aguas residuales producto del proceso de deshilado, lo cual permitirá conocer si los tintes empleados son orgánicos o contienen elementos nocivos para el ambiente; hacer el análisis desde la ecoeficiencia y el ecodiseño para comprobar que las mezclas del nuevo material pueden representar una solución de largo plazo. Asimismo, de continuar con la elaboración de mezclas con deshilado y cualquiera de las matrices poliméricas, es necesario establecer el volumen óptimo de fibra deshilada dentro de dicha matriz para verificar la eficacia de este método para la revalorización de este desperdicio.

Finalmente, como experiencia didáctica es necesario involucrar al diseñador industrial en el proceso de manufactura no solo del producto, sino de los materiales e insumos para que experimente con ellos y que los pueda utilizar para diseñar en el futuro. Esto le permitirá vislumbrar oportunidades para el diseño no solo de productos, sino de sistemas de orden superior.

\section{Agradecimientos:}

Nuestros agradecimientos a Nancy Gabriela Rojas, Sayda Mascareño Lugo, Sandra Rosa Padilla Martínez, Abigail Irene Hermosillo Rodríguez, Ana Emilia León Sánchez, Mariel Vázquez García, Eric Nápoles López, América Lilian Díaz Vergara, Ashley Aida López Moreno, Lauren Juncal López Paredes, Brenda Janette Trujillo Castellanos y Ricardo Juárez Nájera por su entusiasmo y entrega durante el proyecto. Asimismo, agradecemos al PRODEP por su apoyo para la primera etapa de este proyecto. 


\section{Referencias}

Ayala-García, C., Rognoli, V., \& Karana, E. (2017). “Five Kingdoms of DIY-Materials for Design". In EKSIG2017: Alive. Active. Adaptive-International Conference on Experiential Knowledge and Emerging Materials, pp. 222-234. Rotterdam: Het Nieuwe Instituut. Retrieved from http://www.eksig2017.com/proceedings/

Bakker, C., Wever, R., Teoh, C., \& De Clercq, S. (2010). "Designing cradle-to-cradle products: a reality check". International Journal Of Sustainable Engineering, 3(1), 2-8. doi: 10.1080/19397030903395166

Braungart, M. y W. McDonough. (2002). Cradle to Cradle, Remaking the way we make things. 1st ed. Nueva York.

Brydson, J. A. (2000). Plastics Materials. Oxford: Butterworth Heinemann.

Chavan, R. B. (2015). En Roshan, P. (Ed.) Denim Manufacture, finishing and applications. Elsevier.

Gómez, J. F., et al., (2016). “Evaluación de compuestos de PP-residuos de mezclilla para la elaboración de un nuevo material para el diseño", Legado Revista de Arquitectura y Diseño, no. 19, enero-junio.
Gómez, J. F., et al., (2016). “Scrap denim-PP composites as a material for new product design", RDIS Revista Online de la Red Internacional de Investigación en Diseño, vol. 2, no.3, pp. 82-91.

Kopnina, H. (2018). Circular economy and Cradle to Cradle in educational practice. Journal of Integrative Environmental Sciences, 15(1), 123-138. doi: $10.1080 / 1943815 \times .2018 .1471724$

Metcalf, G. (2014). Social systems and design. Tokio: Springer.

Miller, D., \& Woodward, S. (2007). "A Manifesto for the Study of Denim". Social Anthropology/Anthropologie Sociale, 15(3), pp. 335-351.

Rosa, L.A., et al., (2015). "Aprovechamiento de residuos industriales para el diseño y desarrollo de productos para la industria de la moda con un enfoque sostenible". Memorias del Congreso Internacional de Investigación Academia Journals.

Toxopeus, M., de Koeijer, B., \& Meij, A. (2015). Cradle to Cradle: Effective Vision vs. Efficient Practice? Procedia CIRP, 29, 384-389. doi: 10.1016/j.procir.2015.02.068 